\title{
Experimental Study on Enhanced Condensate Recovery by Gas Injection in Yaha Condensate Gas Reservoir
}

\author{
Yiming Wu, ${ }^{1}$ Kun Yao, ${ }^{1}$ Yan Liu, ${ }^{1}$ Xiangyun Li, ${ }^{2}$ Mimi Wu, ${ }^{1}$ Ronghong Cheng, \\ and Bo Wang $\oplus^{3}$ \\ ${ }^{1}$ Research Institute of Exploration and Exploitation, Petro-China Tarim Oilfield Company, Korla 841000, China \\ ${ }^{2}$ CNPC USA Corporation, Beijing 100028, China \\ ${ }^{3}$ Petroleum Systems Engineering, Faculty of Engineering and Applied Science, University of Regina, Regina, \\ Saskatchewan S4S 0A2, Canada
}

Correspondence should be addressed to Bo Wang; wbg373@uregina.ca

Received 8 August 2021; Revised 8 September 2021; Accepted 20 September 2021; Published 14 October 2021

Academic Editor: Jinjie Wang

Copyright (c) 2021 Yiming Wu et al. This is an open access article distributed under the Creative Commons Attribution License, which permits unrestricted use, distribution, and reproduction in any medium, provided the original work is properly cited.

A condensate gas reservoir is an important special oil and gas reservoir between oil reservoir and natural gas reservoir. Gas injection production is the most commonly used development method for this type of gas reservoir, but serious retrograde condensation usually occurs in the later stages of development. To improve the recovery efficiency of condensate oil in the middle and late stages of production of a condensate gas reservoir, a gas injection parameter optimization test study was carried out, taking the Yaha gas condensate reservoir in China as an example. On the premise that the physical experimental model and key parameters met the actual conditions of the formation, the injection method, injection medium, injectionproduction ratio, and other parameters of the condensate gas reservoir were studied. Research on the injection method showed that the top injection method had a lower gas-oil ratio and higher condensate oil recovery. The study of injection medium showed that the production effect of carbon dioxide $\left(\mathrm{CO}_{2}\right)$ injection was the best injection medium, and the maximum recovery rate of condensate oil was $95.11 \%$. The injection-production ratio study showed that the injection-production ratio was approximately inversely proportional to the recovery factor of condensate gas and approximately proportional to the recovery factor of condensate oil. When the injection-production ratio was $1: 1$, the maximum recovery rate of condensate oil was $83.31 \%$. In summary, in the later stage of gas injection development of the Yaha condensate gas reservoir, it was recommended to choose the development plan of $\mathrm{CO}_{2}$ injection at the top position with an injection-production ratio of $1: 1$. This research can not only provide guidance for the later formulation of gas injection plans for Yaha condensate gas reservoirs but also lay a foundation for the research of gas injection migration characteristics of other condensate gas reservoirs.

\section{Introduction}

With the continuous improvement of the level of exploration technology and exploration degree at home and abroad, the proportion of condensate gas reservoirs discovered is increasing year by year. Therefore, condensate gas fields occupy a particularly important position in the development of gas fields in the world. Condensate gas reservoir is different from ordinary oil reservoir or gas reservoir; it has the dual characteristics of oil reservoir and gas reservoir [1]. Due to the special fluid phase characteristics, its extraction technology and development difficulty are much more com- plicated than general gas reservoirs and oil reservoirs [2-4]. During the development of condensate gas reservoirs, when the reservoir pressure is lower than the dew point pressure, serious retrograde condensation will occur in the reservoir. In other words, condensate oil will separate out of the gas phase and accumulate in a large amount in the nearwellbore zone, which will block the original seepage pores, thereby reducing the final recovery rate of condensate gas and condensate oil [5-9].

Compared with natural gas, condensate oil has extremely high economic value. At present, there are two main methods to improve the recovery rate of condensate 
TABLE 1: Condensate gas reservoir parameters and content.

\begin{tabular}{|c|c|c|c|}
\hline Category & Parameter & Numerical & Unit \\
\hline \multirow{4}{*}{ Condensate gas properties } & Density & $0.63 \sim 0.67$ & $\mathrm{~kg} / \mathrm{m}^{3}$ \\
\hline & $\mathrm{CO}_{2}$ & $<1$ & $\%$ \\
\hline & $\mathrm{N}_{2}$ & $3 \sim 8$ & $\%$ \\
\hline & $\mathrm{C}_{1}$ & 85 & $\%$ \\
\hline \multirow{6}{*}{ Condensate oil properties } & Density & $0.78 \sim 0.83$ & $\mathrm{~kg} / \mathrm{m}^{3}$ \\
\hline & Freezing point & $9 \sim 36$ & ${ }^{\circ} \mathrm{C}$ \\
\hline & Sulfur content & $<0.12$ & $\%$ \\
\hline & Wax content & $5.74 \sim 13.77$ & $\%$ \\
\hline & Wax-off point & $9 \sim 20.5$ & ${ }^{\circ} \mathrm{C}$ \\
\hline & Gum and asphalt content & Low content & - \\
\hline \multirow{2}{*}{ Formation water properties } & Density & $1.08 \sim 1.44$ & $\mathrm{~kg} / \mathrm{m}^{3}$ \\
\hline & Salinity & $137752 \sim 214209$ & $\mathrm{mg} / \mathrm{l}$ \\
\hline \multirow{3}{*}{ Gas reservoir properties } & Condensate oil content & $600 \sim 700$ & $\mathrm{~g} / \mathrm{m}^{3}$ \\
\hline & Maximum reverse condensation pressure & $25 \sim 30$ & $\mathrm{MPa}$ \\
\hline & Maximum reverse condensate volume & 30 & $\%$ \\
\hline
\end{tabular}

TABLE 2: Geological characteristic parameters of condensate gas reservoirs.

\begin{tabular}{lcccccccc}
\hline $\begin{array}{l}\text { Reservoir } \\
\text { location }\end{array}$ & $\begin{array}{c}\text { Thickness } \\
(\mathrm{m})\end{array}$ & $\begin{array}{c}\text { Average } \\
\text { thickness } \\
(\mathrm{m})\end{array}$ & $\begin{array}{c}\text { Porosity } \\
(\%)\end{array}$ & $\begin{array}{c}\text { Average } \\
\text { porosity } \\
(\%)\end{array}$ & $\begin{array}{c}\text { Permeability } \\
\left(10^{-3} \mu \mathrm{m}^{2}\right)\end{array}$ & $\begin{array}{c}\text { Average } \\
\text { permeability } \\
\left.\mu^{2}\right)\end{array}$ & $\begin{array}{c}\text { Multiple of } \\
\text { permeability }\end{array}$ & $\begin{array}{c}\text { Average multiple } \\
\text { of permeability }\end{array}$ \\
\hline Paleogene & $0.2 \sim 3.4$ & 0.9 & $16.4 \sim 21.21$ & 17.9 & $120.7 \sim 2257.7$ & 641.4 & $2.6 \sim 7.6$ & 3.4 \\
Cretaceous & $0.2 \sim 1.6$ & 0.5 & $11.2 \sim 18.34$ & 16 & $33.6 \sim 233.9$ & 89.3 & $2.6 \sim 6.2$ & 3.4 \\
\hline
\end{tabular}

TABLE 3: Experimental program.

\begin{tabular}{|c|c|c|c|c|}
\hline $\begin{array}{l}\text { Experiment } \\
\text { category }\end{array}$ & $\begin{array}{l}\text { Experiment } \\
\text { grouping }\end{array}$ & Experiment content & $\begin{array}{l}\text { Experimental } \\
\text { program }\end{array}$ & Experimental parameters \\
\hline \multirow{3}{*}{1} & 1 & \multirow{3}{*}{ Gas injection method } & Side gas injection & \\
\hline & 2 & & $\begin{array}{l}\text { Bottom gas } \\
\text { injection }\end{array}$ & \\
\hline & 3 & & Shaft gas injection & $\begin{array}{c}\text { Temperature: } 137.8^{\circ} \mathrm{C} \\
\text { Pressure: } 54.94 \mathrm{MPa}\end{array}$ \\
\hline \multirow{3}{*}{2} & 4 & \multirow{3}{*}{ Gas injection medium } & $\mathrm{CH}_{4}$ & Initial pressure: $60 \mathrm{MPa}$ \\
\hline & 5 & & On-site gas & Injection speed: $0.2 \mathrm{ml} / \mathrm{min}$ \\
\hline & 6 & & $\mathrm{CO}_{2}$ & Injection and mining method: one injection and one \\
\hline \multirow{3}{*}{3} & 7 & \multirow{3}{*}{$\begin{array}{l}\text { Injection-production } \\
\text { ratio }\end{array}$} & $0.5: 1$ & mining \\
\hline & 8 & & $0.75: 1$ & \\
\hline & 9 & & $1: 1$ & \\
\hline
\end{tabular}

oil: one is continuous depletion development, and the other is gas injection to maintain pressure. In the process of depletion mining, natural energy is gradually released and pressure-free replenishment system. The injection pressure-holding mining method is to inject gaseous media into the reservoir to supplement the formation energy to reduce retrograde condensate damage [10-12]. Scholars at home and abroad have conducted in-depth discussions on these two methods. For example, Jingsong et al. [13] used reservoir numerical simulation methods to analyze the sensitivity of injection parameters for cyclic gas injection devel- opment of condensate gas reservoirs, focusing on evaluating the impact of injection methods, gas injection timing, and injection-production ratios on the development effects of condensate gas reservoirs. The results show that for condensate gas reservoirs rich in condensate oil and with a large ground pressure difference, the development effect of the top cycle gas injection after depletion development to the dew point pressure is better. Dong et al. [14] took the Yaha-5 condensate gas reservoir as an example. The three production methods of exhaustion, water injection, and gas injection were compared and analyzed by component 


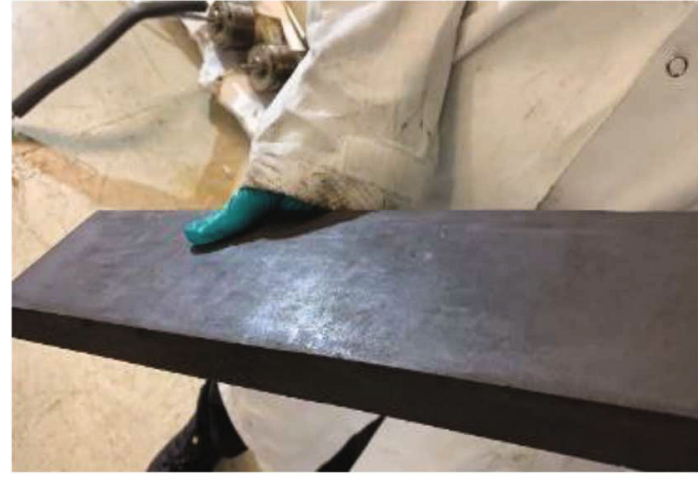

Figure 1: Schematic diagram of the core sample.

numerical simulation. The research results show that water injection and gas injection are better than depletion mining, and gas injection is better than water injection. In particular, the large well spacing gas injection development method can not only delay gas channeling and improve gas injection efficiency but also greatly increase the degree of condensate oil recovery. Lu [15] took Dalaoba No. 2 condensate gas reservoir with high condensate oil content as an example. The gas injection state and seepage mechanism are analyzed, and pilot experiments are carried out. The research results show that when entering the middle and late stages of mining, the formation pressure drops faster, the retrograde condensation phenomenon is serious, and the formation edge water is active. Therefore, it is not appropriate to choose depletion mining or water injection development in the middle and late stages of mining, and gas injection and pressuremaintaining development methods should be considered. $\mathrm{Lu}$ [16] made a comparative analysis and summary of the current recovery methods of condensate gas fields and discussed the strategies for optimizing gas injection production methods. The research results indicate that the optimization of production system, gas injection method, and stop injection time should be strengthened in the development process to improve the recovery rate of condensate gas fields. Through research, it can be found that the depletion mining cost is low and the process is relatively simple, so this method is widely used in the development of condensate gas reservoirs. The method of gas injection to maintain pressure is the most important method to improve the recovery of condensate, especially for condensate gas reservoirs with high condensate oil content, because injecting gas into the reservoir can not only increase the formation pressure in the retrocondensation zone but also reduce the antievaporation effect of the condensate oil. Therefore, the condensate oil is more easily produced. If pressure-holding mining is not carried out, the loss of condensate oil will even reach more than $60 \%$ to $70 \%$ of the original reserves [17-20].

Following the above research, most foreign scholars have conducted in-depth discussions on various issues under the conditions of gas injection. Through literature research, the methods of research problems can be roughly divided into two categories: one is reservoir numerical simulation methods, and the other is experimental analysis and research. For numerical simulation research methods, $\mathrm{He}$
TABLE 4: Laboratory compound condensate gas composition table.

\begin{tabular}{lcccc}
\hline Component & $\begin{array}{c}\text { Actual } \\
\text { Mole fraction } \\
(\mathrm{mol} \%)\end{array}$ & \multicolumn{2}{c}{$\begin{array}{c}\text { Laboratory } \\
\text { Mole fraction (mol\%) }\end{array}$} \\
\hline $\mathrm{N}_{2}$ & $3.26 \%$ & $79.85 \%$ & $\mathrm{CH}_{4}$ & $81.44 \%$ \\
$\mathrm{CH}_{4}$ & $76.59 \%$ & & \\
$\mathrm{CO}_{2}$ & $0.62 \%$ & & \\
$\mathrm{C}_{2} \mathrm{H}_{6}$ & $8.90 \%$ & & \\
$\mathrm{C}_{3} \mathrm{H}_{8}$ & $1.83 \%$ & & & \\
$\mathrm{iC}_{4}$ & $0.48 \%$ & & & \\
$\mathrm{nC}_{4}$ & $0.71 \%$ & $13.81 \%$ & & \\
$\mathrm{iC}_{5}$ & $0.34 \%$ & & & \\
$\mathrm{nC}_{5}$ & $0.34 \%$ & & & \\
$\mathrm{C}_{6}$ & $0.59 \%$ & & & \\
$\mathrm{C}_{7}$ & $1.10 \%$ & & & \\
$\mathrm{C}_{8}$ & $1.12 \%$ & & & \\
$\mathrm{C}_{9}$ & $0.59 \%$ & $6.34 \%$ & On-site condensate & $4.90 \%$ \\
$\mathrm{C}_{10}$ & $0.48 \%$ & & & \\
$\mathrm{C}_{11}{ }^{+}$ & $3.05 \%$ & & & \\
\hline
\end{tabular}

et al. [21] took the condensate gas reservoir in the southern part of Rangnar A as an example, applied phase equilibrium theory and reservoir numerical simulation technology, and studied the mechanism and effect of $\mathrm{CO}_{2}$ huff and puff to increase gas well condensate production. The research results show that when the amount of $\mathrm{CO}_{2}$ injected is small, the capacity of $\mathrm{CO}_{2}$ vaporization and condensate is limited. To ensure the effect of $\mathrm{CO}_{2}$ huff and puff to increase oil, the periodic injection of $\mathrm{CO}_{2}$ should exceed $500 \times 10^{4} \mathrm{~m}^{3}$. Hassan et al. [22] used thermochemical treatment methods to treat the near-well zone and used reservoir simulation methods to simulate oil and gas recovery. The research results show that this method can significantly improve oil and gas recovery, and the main reason for this phenomenon is that this method can reduce capillary pressure and viscosity of condensate oil well. Wan and Mu [23] took the Eagle Ford shale gas condensate gas reservoir as an example, used numerical simulation to study the effect of carbon dioxide steam huff and puff injection on slowing down the accumulation of condensate around the induced fractures, and conducted in-depth discussions on the molecular scale. The research results show that the use of $\mathrm{CO}_{2}$ huff and puff gas injection is more conducive to improving the recovery of rich condensate oil. Jiang and Younis [24] used a multicomponent molecular simulation method to perform a numerical analysis on the enhanced oil recovery of carbon dioxide steam huff and puff in a complex fractured condensate gas reservoir. On this basis, several design elements such as the number of cycles and the length of the injection period in the steam stimulation process are briefly studied. For experimental analysis and research methods, Feng et al. [25] took Sulige tight sandstone condensate gas reservoir in Ordos Basin as an example. Based on the results of PVT phase experiments, core gas injection displacement experiments were carried out. An in-depth study of continuous gas 


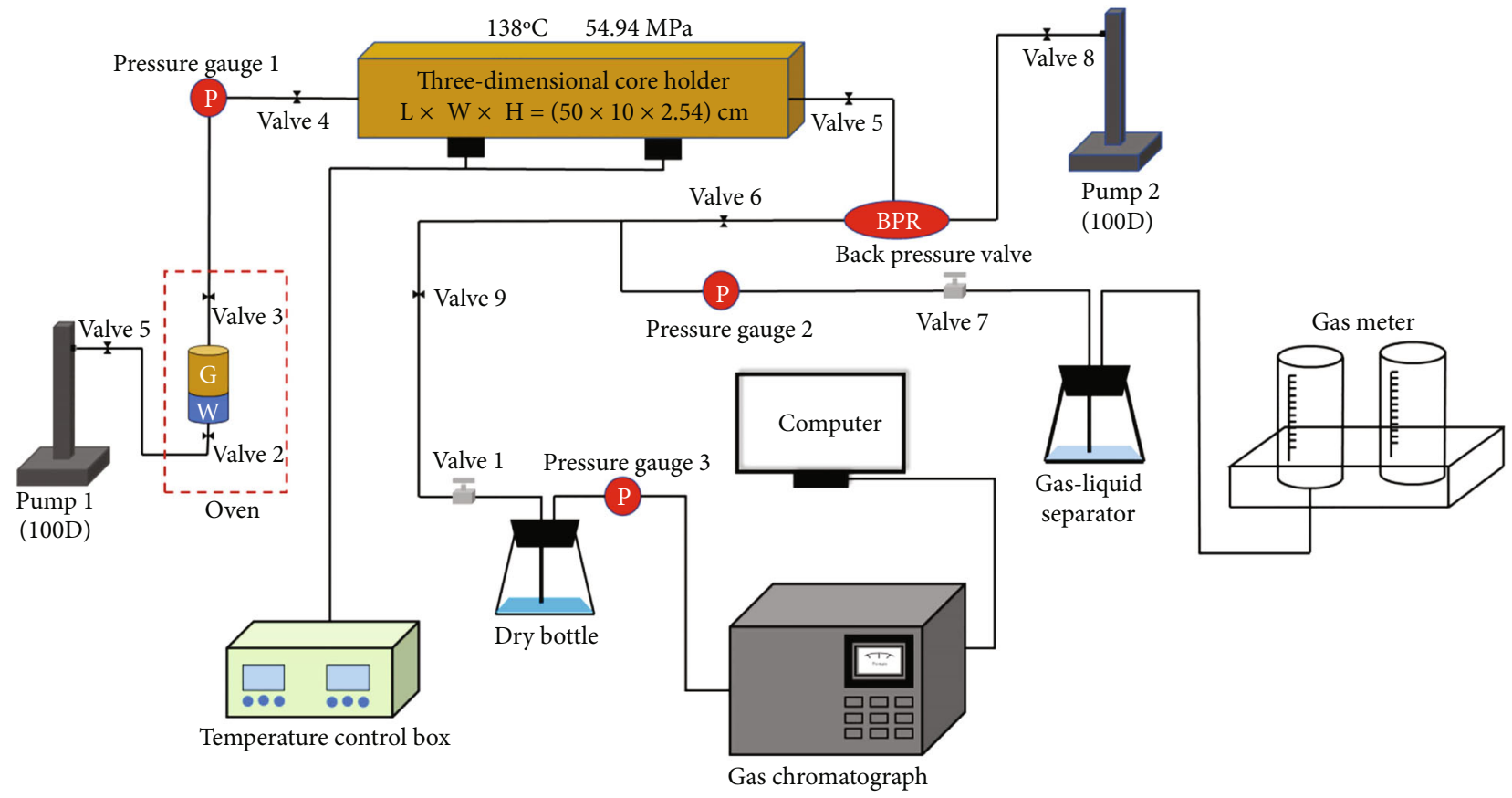

(a) Schematic diagram of theoretical model

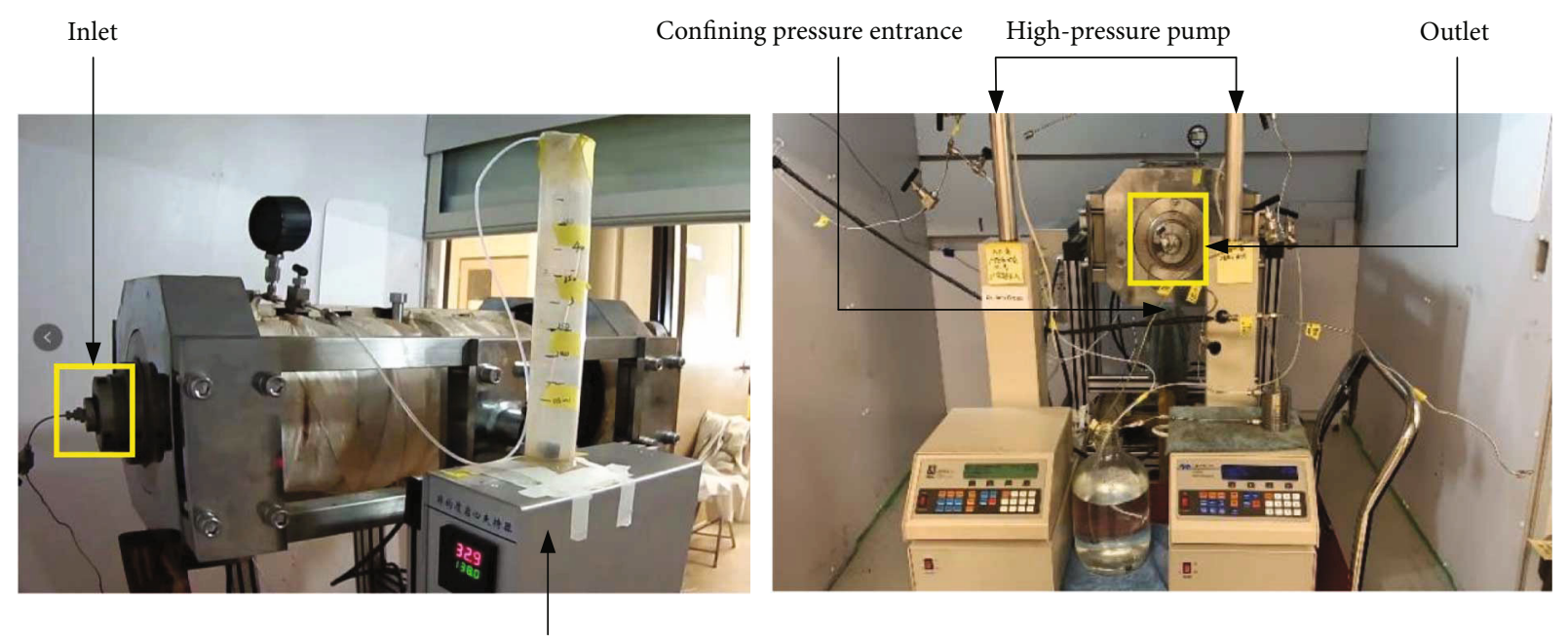

Temperature control box

(b) Schematic diagram of actual device

Figure 2: Schematic diagram of experiment setup.

injection, gas injection huff and puff and pulse gas injection condensate recovery degree, and the change characteristics of average condensate oil saturation in the core was conducted, and then, the best gas injection method to improve the condensate oil recovery was optimized. Yuan et al. [26] took the Zhongyuan Oilfield high-pressure and highsaturation condensate gas reservoir as the research object. The laboratory test study of methane $\left(\mathrm{CH}_{4}\right)$ injection for enhanced oil recovery in high-pressure and high-saturated condensate gas reservoirs has been carried out. The research results show that for high-pressure and high-saturation condensate gas fields, first depletion production to a certain extent and then gas injection can also achieve higher recovery. Hou [27] used the fully visible mercury-free high-temperature and high-pressure multifunctional formation fluid PVT analyzer to conduct an experimental study on the phase behavior of an offshore high-carbon dioxide condensate gas well. The experimental results show that the higher the $\mathrm{CO}_{2}$ content, the higher the condensate oil-gas-oil ratio, the greater the condensate density, the higher the condensate dew point pressure, the larger the relative volume of condensate, and the smaller the amount of reverse condensate. Therefore, carbon dioxide injection can improve the recovery efficiency of condensate oil. For this conclusion, some scholars have also verified this view $[8,28]$. 


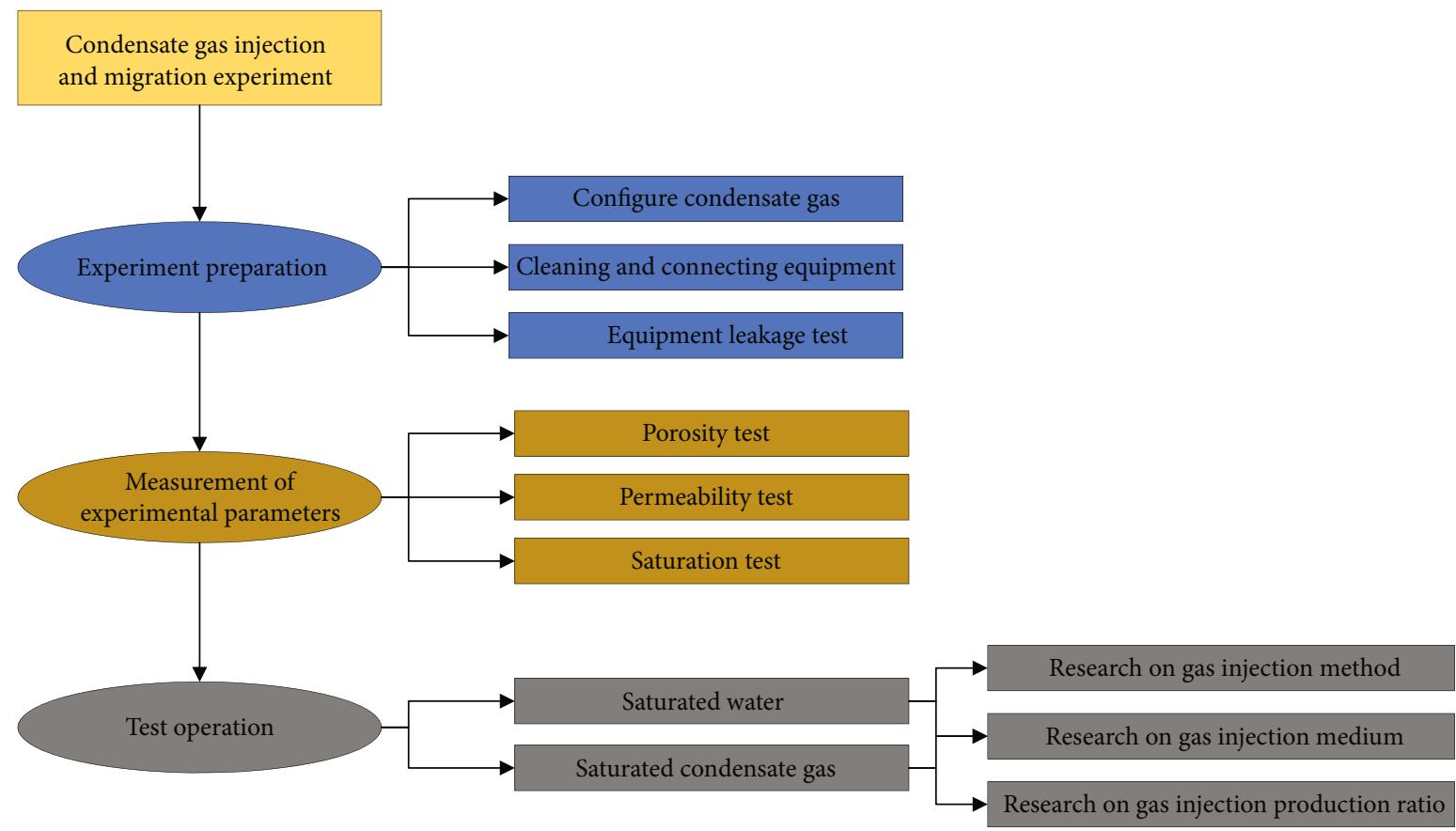

FIgURE 3: Experiment procedure flowchart.

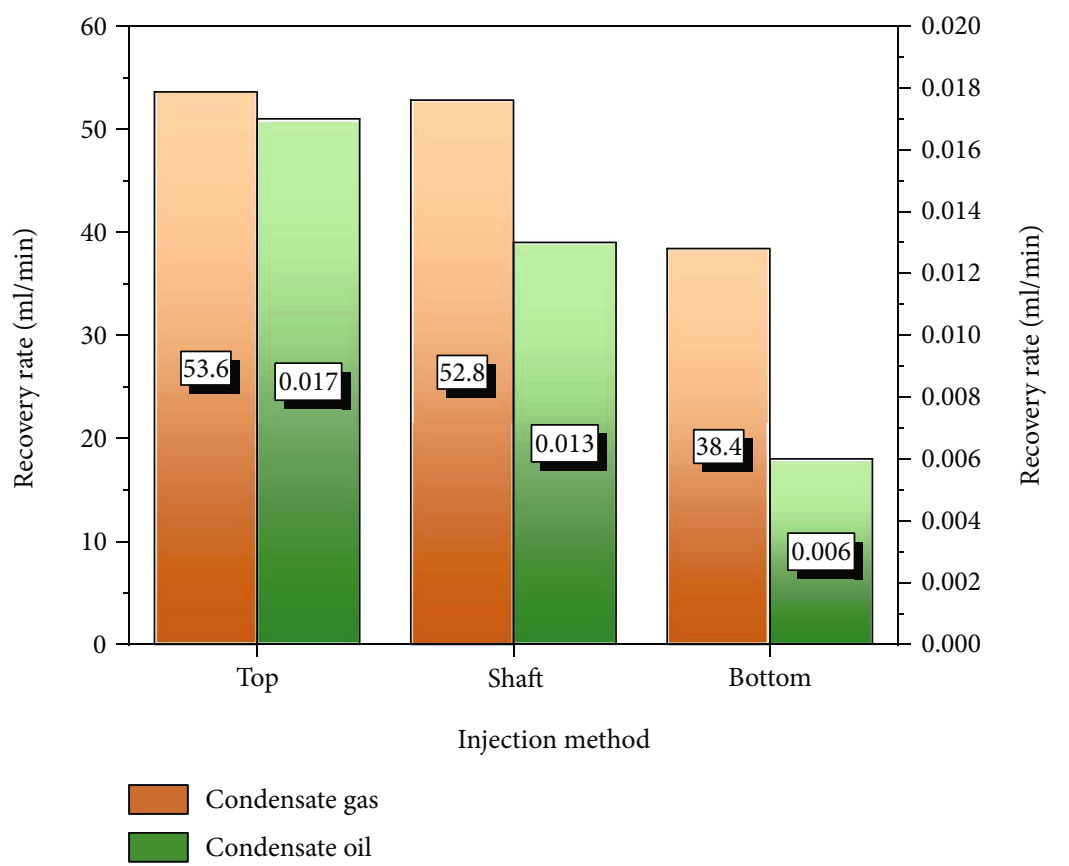

FIgURE 4: Histogram of the average recovery rate of different gas injection methods.

According to the above analysis, the research on improving the condensate gas reservoir by gas injection and maintaining pressure mainly uses numerical simulation methods and focuses on the elimination of retrograde condensate theory and complex phase transition issues. For experimental research, most of the research focuses on the selection of gas injection medium and the discussion of gas injection methods. There are few reports on the analysis of key parameters and variables in the development method of gas injection and pressure maintenance. However, studying the migration mechanism and law of injected gas has become an urgent problem to be solved to improve the recovery rate of gas injection in condensate gas reservoirs. In-depth study of the migration law of injected gas in condensate gas reservoirs is the key to increasing gas injection utilization and improving development effects.

To sum up, to research the gas injection migration characteristics of the Yaha condensate gas reservoir and improve 


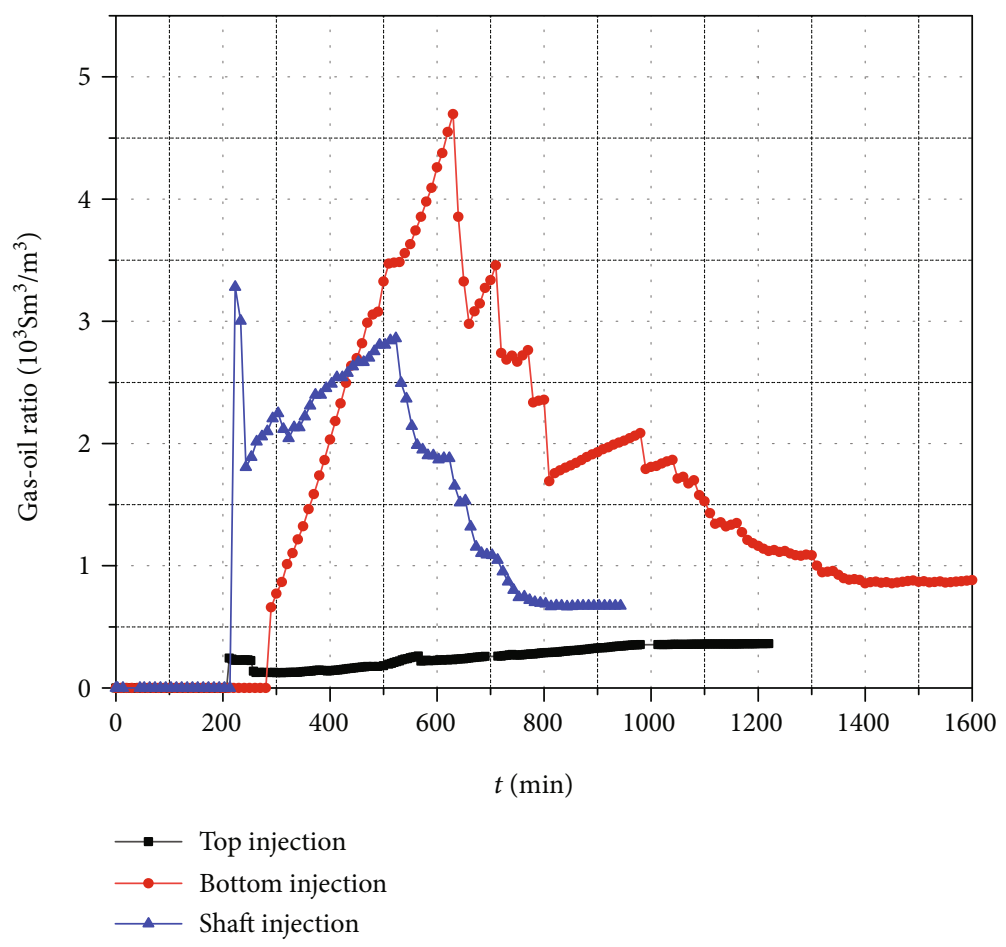

FIGURE 5: Gas-oil ratio curves produced by different gas injection methods.

the oil recovery in the later stage of the oilfield development, this research is based on the oilfield site and conducted indoor physical model experiments. First, the gas reservoir and geological profile are described. Then, the condensate gas injection and migration experiments are carried out. Finally, by changing the gas injection method, gas injection medium, and injection-production ratio, the influence of various variables on the enhanced oil recovery of condensate gas injection was clarified. This research can not only provide guidance for the later formulation of gas injection plans for Yaha condensate gas reservoirs but also lay a foundation for the research of gas injection migration characteristics of other condensate gas reservoirs.

\section{Gas Reservoir Overview and Geological Characteristics}

2.1. Condensate Gas Reservoir Overview. The Yaha condensate gas reservoir is located in Kuqa County, Xinjiang Uygur Autonomous Region, China, with an altitude of $967 \sim 1033 \mathrm{~m}$. This gas reservoir is the largest condensate gas reservoir developed by cyclic gas injection in China. The gas reservoir structure is located on the Yaha fault structural belt in the Tarim Basin, distributed from northeast to southwest. The formation pressure of the gas reservoir is $53 \sim 56 \mathrm{MPa}$, the ground pressure difference is $2 \sim 4 \mathrm{MPa}$, and the condensate oil content is relatively high $\left(500 \sim 5600 \mathrm{~g} / \mathrm{m}^{3}\right)$. It is a high-pressure condensate gas reservoir close to saturation with high condensate oil content. The condensate oil in the gas reservoir has low density, low viscosity, low content of colloidal asphalt, high wax content, and high freezing point. The $\mathrm{H}_{2} \mathrm{~S}$ content in the con- densate gas is very small, the $\mathrm{CO}_{2}$ content is low, and the $\mathrm{N}_{2}$ content is high. The specific conditions of condensate gas reservoirs are shown in Table 1.

2.2. Geological Characteristics. The Yaha condensate gas reservoir is vertically divided into two gas layer groups: the bottom sandstone of the Paleogene and the top sandstone of the Cretaceous (Table 2). The above two condensate gas layers are both massive bottom water condensate gas reservoirs rich in condensate oil. The Paleogene gas reservoir is dominated by gray-brown fine sandstone, followed by coarse sandstone, medium sandstone, and gypsum sandstone. The Paleogene gas reservoir has low shale content and belongs to a chemically cemented reservoir. The Cretaceous gas reservoir is brown-red and brown siltstone, medium-fine sandstone with brown-red mudstone, and argillaceous siltstone. The Cretaceous gas reservoir is a lime mud cemented reservoir. Therefore, the Yaha condensate gas reservoir has the characteristics of deep burial, high formation pressure, small ground pressure difference, high condensate oil content, maximum retrograde condensate pressure, and high maximum retrograde condensate liquid volume.

\section{Experiment Overview}

Based on the actual geology of the Yaha condensate gas reservoir, this experiment conducted a study on the characteristics of gas injection migration. Under the condition that the core properties, fluid properties, initial conditions, and experimental procedures remain unchanged, the gas injection method, gas injection medium, and injectionproduction ratio are changed, and the gas injection scheme 


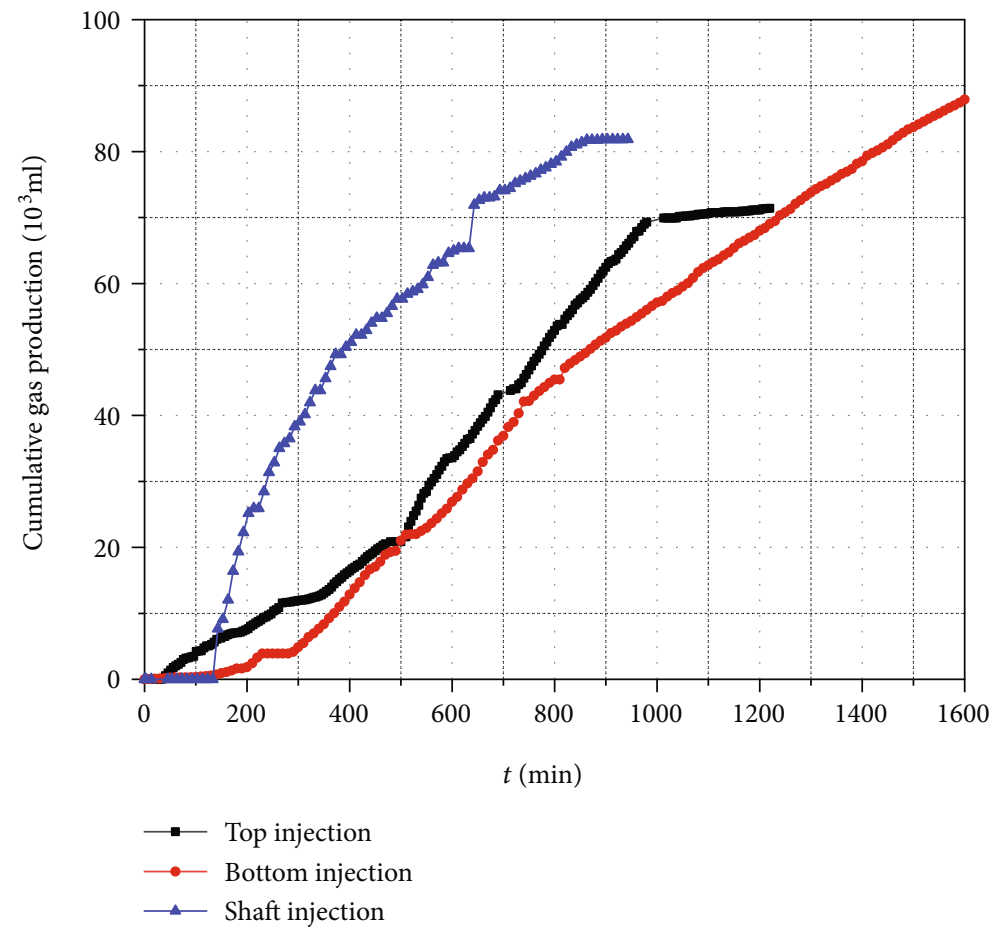

(a) Cumulative gas production curve

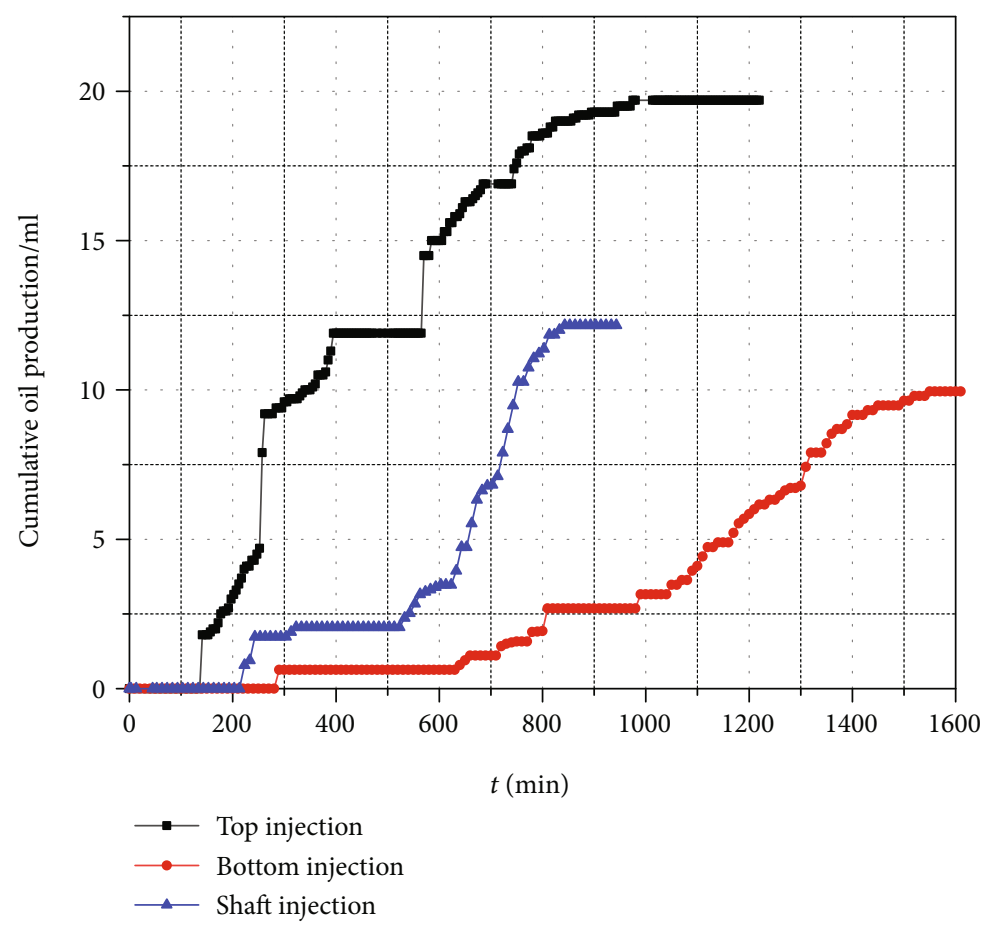

(b) Cumulative oil production curve

Figure 6: Cumulative production curve of different injection methods.

is optimized to improve the recovery effect of the condensate gas reservoir. The experiment designed 3 types ( 9 groups) of programs; the specific conditions are shown in Table 3.

3.1. Experiment Materials. The core used in the experiment was provided by China Chengdu Core Technology Com- pany, as shown in Figure 1. The core sample is a homogeneous low-permeability core slab manufactured artificially, and its size is $L \times W \times H=50 \times 10 \times 2.54(\mathrm{~cm})$.

Based on the actual situation of the oil field, using CMG WinProp software, according to the mole fraction, the condensate gas composition and dosage used in the experiment 


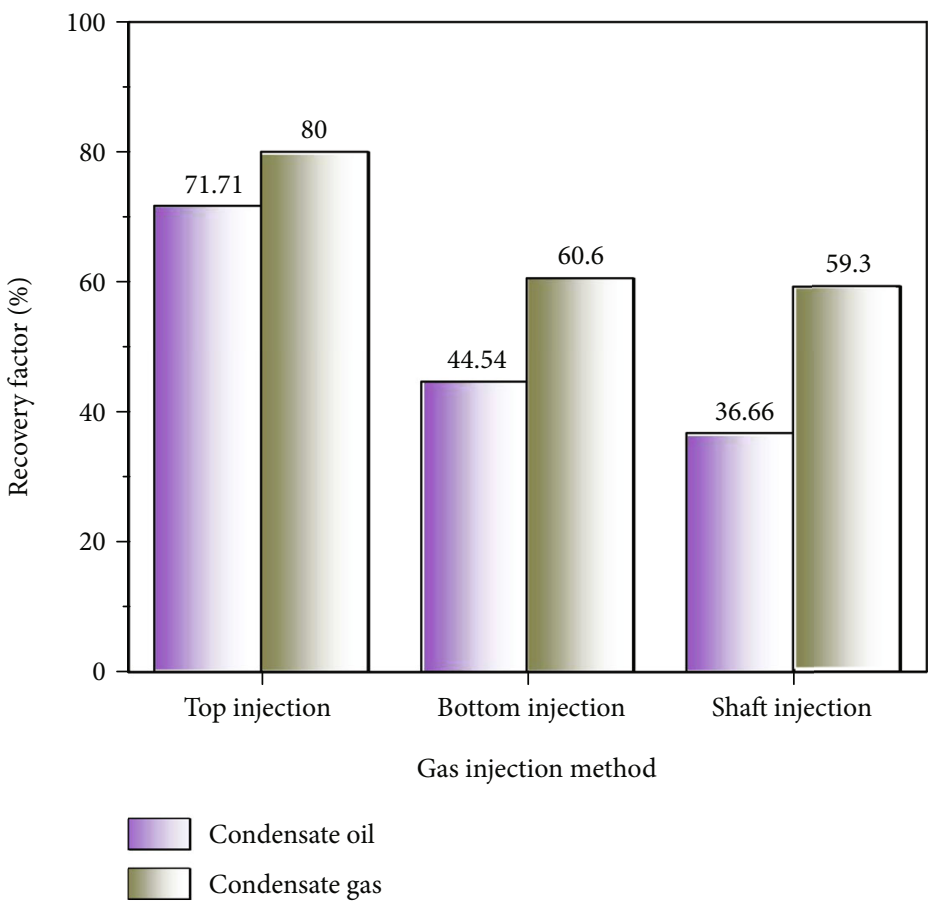

Figure 7: Histogram of final recovery factor of different gas injection methods.

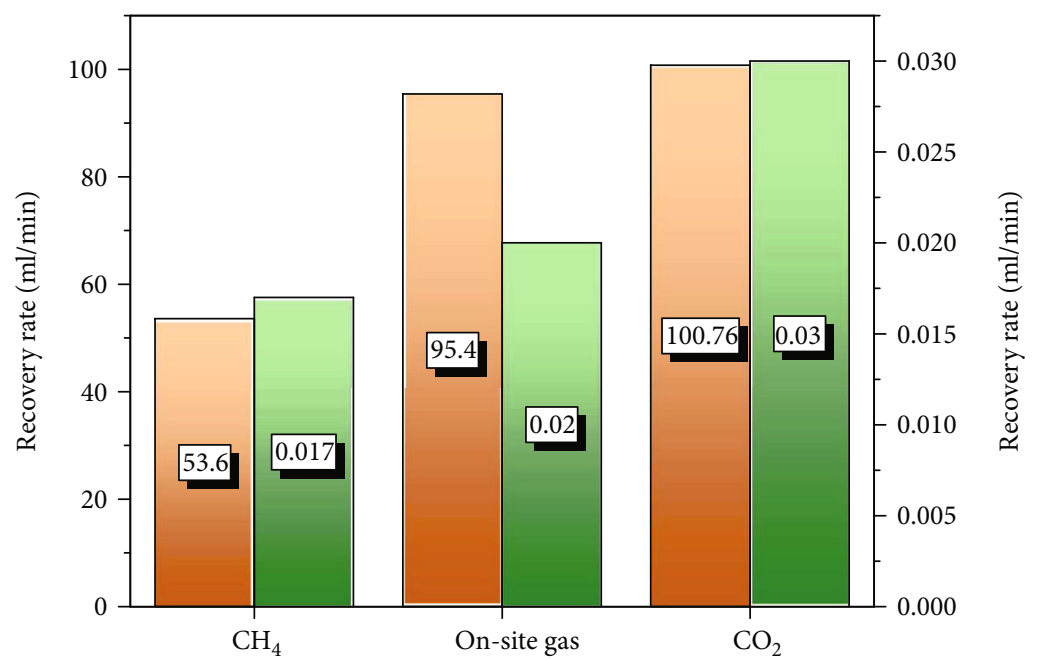

Injection medium

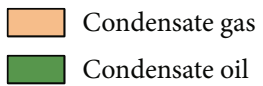

FIGURE 8: Histogram of average recovery speed of different gas injection media.

were determined. The properties of the actual fluid on site and the fluid configured in the laboratory are shown in the table. It can be seen from Table 4 that the actual condensate gas in the reservoir is mainly composed of methane (76.59\%), ethane (83.9\%), and macromolecular liquid hydrocarbon compounds. To ensure the feasibility and accuracy of the experiment, similar condensate gas components are used for coordination and modulation. It is finally deter- mined that the condensate gas composition is methane $(81.44 \%)$, ethane $(13.66 \%)$, and condensate oil $(4.90 \%)$. The gas raw materials prepared by the condensate gas are provided by Praxair Canada Inc., and the purity is as high as $99.99 \%$.

3.2. Experiment Setup. The experiment setup of the gas injection migration experiment in the condensate gas reservoir is 


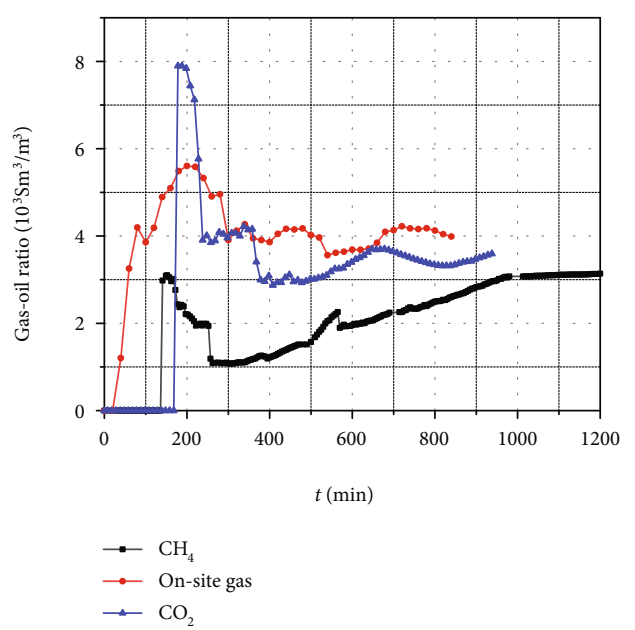

FIGURE 9: Curves of gas-oil ratio produced by different gas injection media.

shown in Figure 2. Among them, Figure 2(a) is a theoretical schematic diagram, and Figure 2(b) is an actual device diagram.

It can be seen from Figure 2 that the physical simulation experiment in this research mainly includes a threedimensional core holder, a high-temperature and highpressure intermediate container, and a high-pressure injection pump. Among them, the three-dimensional core holder is selected from Haian Petroleum Instrument Factory, with a maximum pressure of $70 \mathrm{MPa}$ and a maximum temperature of $200^{\circ} \mathrm{C}$. In order to meet the needs of the experiment, it uses fluorine rubber special core rubber sleeve inside. Two types of high-temperature and high-pressure resistant intermediate containers are selected, $206 \mathrm{ml}$ and $1000 \mathrm{ml}$, and their maximum pressure is $70 \mathrm{MPa}$ and the maximum temperature is $200^{\circ} \mathrm{C}$. The high-pressure injection pump adopts ISCO-100DX model produced by Teledyne, USA, and the maximum injection pressure is $71 \mathrm{MPa}$.

3.3. Experiment Procedure. In this research, the experiment includes three parts: experiment preliminary preparation, experimental parameter determination, and test operation. The specific situation is as shown in Figure 3.

In the stage of experiment preliminary preparation, the condensate gas used in the experiment is configured according to the actual parameters in the oilfield reservoir. Then, the experimental platform is assembled according to the experimental plan, and the ethanol solution is used to clean and leak test it.

In the stage of experimental parameter determination, deionized water is used to test the porosity of core samples. The porosity is calculated based on the ratio of water absorption to model volume. Then, the permeability test of the core sample is carried out. The permeability is calculated according to the water injection speed and pressure difference using Darcy's law. Finally, we vacuum the model and wait for the formal test.

In the test operation stage, the core saturated water treatment is performed according to the oilfield geological data, and the initial irreducible water saturation is $30 \%$. Then, the core is treated with saturated condensate gas. Finally, the physical model is used to study the characteristics of gas injection migration in condensate gas reservoirs. By changing the gas injection method, gas injection medium, and injection-production ratio, the optimal condensate gas injection program is further optimized and designed.

\section{Results and Discussion}

4.1. Gas Injection Method. The use of different gas injection methods in condensate gas reservoirs will have a serious impact on the recovery rate and recovery rate of condensate gas and condensate oil. To study the best gas injection method for the Yaha condensate gas reservoir, shaft injection, top injection, and bottom injection were selected for comparative analysis. The experimental gas injection medium is methane, and the injection-production ratio is $0.5: 1$. Other specific experimental parameters are shown in Table 3.

The average recovery rate of condensate gas and condensate oil for different gas injection methods is shown in Figure 4.

It can be seen from Figure 4 that for the average recovery rate of condensate gas, the effects of top gas injection and shaft gas injection are approximately the same, but both are much greater than bottom gas injection. For the recovery rate of condensate oil, the top gas injection has the best effect, with a recovery rate of 0.017 , followed by shaft gas injection with a recovery rate of 0.013 , and finally bottom gas injection with a recovery rate of 0.006 . Therefore, through comparative analysis, it can be known that whether it is the average recovery rate of condensate gas or the average recovery rate of condensate oil, the top gas injection method is the best choice.

To analyze the recovery rate of condensate gas and condensate oil during the entire recovery process, the production gas-oil ratio curves of three different injection methods are drawn, as shown in Figure 5.

It can be seen from Figure 5 that the production gas-oil ratio of the top gas injection method is always the lowest, so the condensate recovery effect of this method is the best. The production gas-oil ratio curves of the shaft gas injection method and the bottom gas injection method roughly show a trend of rising first and then falling. This shows that in the early stage of production, the condensate gas recovery effect of this gas injection method is better. And in the later stage of production, the recovery effect of condensate gas gradually declines, and the recovery effect of condensate oil gradually strengthens, but the effect of condensate oil in the entire production cycle is still lower than that of the top gas injection method.

The cumulative production curves of condensate gas and condensate oil for different gas injection methods are shown in Figure 6.

It can be seen from Figure 6(a) that under the same production pressure, the cumulative gas production curves of the three gas injection methods are quite different, but they all show a gradually increasing trend. In the early stage of 


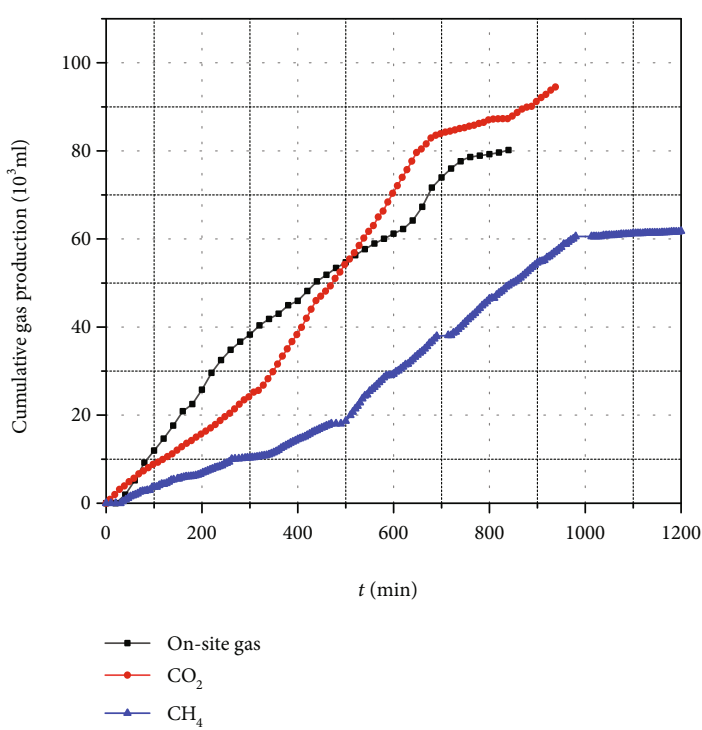

(a) Cumulative gas production curve

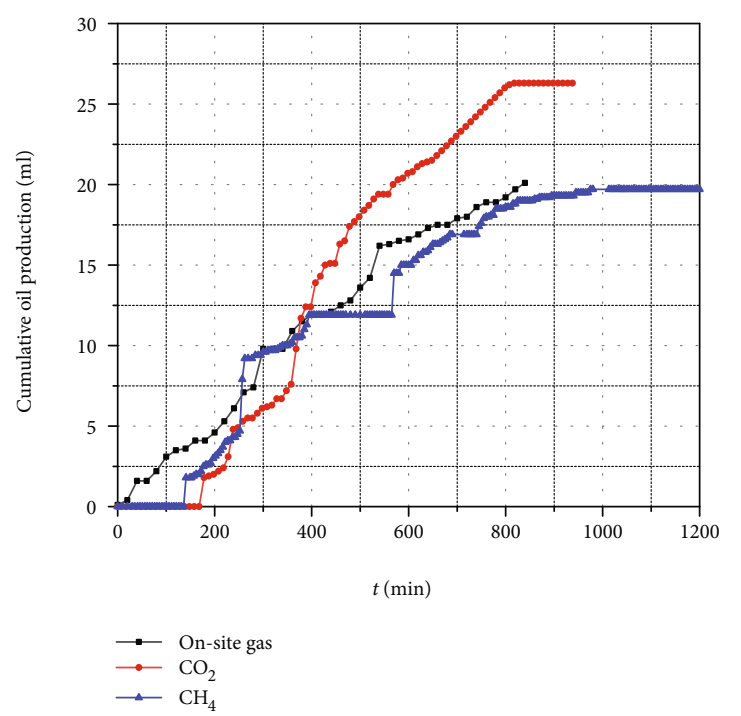

(b) Cumulative oil production curve

Figure 10: Cumulative production curve of different injection media.

gas production $(t<500 \mathrm{~min})$, the shaft gas injection has the fastest gas production speed, followed by bottom gas injection, and top gas injection has the slowest gas production speed. In the middle stage of gas production $(t=500$ $\sim 900 \mathrm{~min}$ ), the gas production rate of shaft gas injection rises rapidly, which is roughly the same as the gas production rate of shaft gas injection, and the gas production rate of bottom gas injection is the slowest. In the later stage of gas production $(t>900 \mathrm{~min})$, the axial gas injection method reached the peak point first, with a peak value of about $80 \times 10^{3} \mathrm{ml}$, followed by the top gas injection method, with a peak value of about $70 \times 10^{3} \mathrm{ml}$. Although the bottom gas injection method has a slower gas production rate, the gas production time is the longest, with a peak value of about $90 \times 10^{3} \mathrm{ml}$.

It can be seen from Figure 6(b) that under the same production pressure, the cumulative oil production curves of the three different gas injection methods are quite different, but the overall trend is gradually increasing. In the early stage of oil production $(t<400 \mathrm{~min})$, the oil production rate and cumulative oil production of the top gas injection method increase rapidly, and they are much higher than the other two gas injection methods. In the mid-stage $(t=400$ $\sim 800 \mathrm{~min}$ ); although the oil production rate of the shaft gas injection method increases rapidly, the cumulative oil production is still much smaller than the top gas injection method, and the bottom gas injection method has the lowest oil production rate and oil production. In the later stage ( $t>800 \mathrm{~min}$ ), the shaft gas injection method stops oil production at the earliest time, and the cumulative oil production finally stabilizes around 12.33 . The second is the top gas injection method, which stops oil production after the mining time is about 1200 minutes, and the final oil production stabilizes around 19.78. The last to stop oil production is the bottom gas injection method. Although this method has the longest cumulative oil production time, the cumulative oil production is the smallest, and the final oil produc-

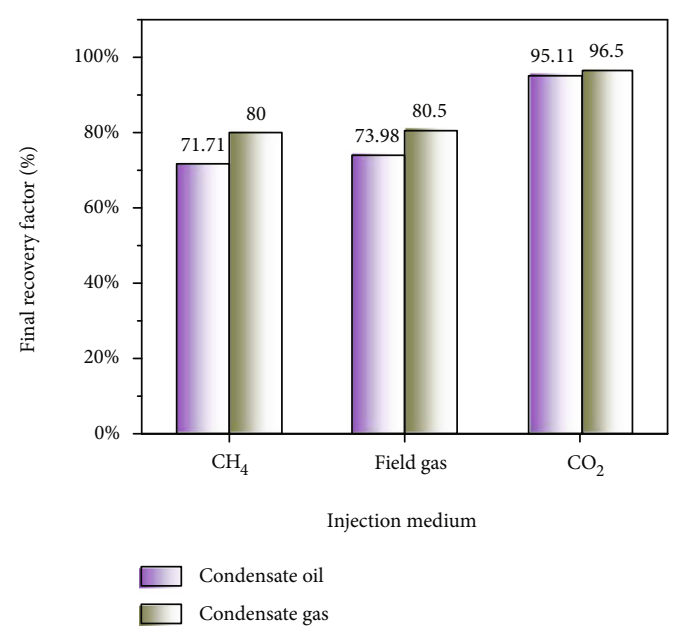

FIgURE 11: Histogram of the final recovery factor with different injection media.

tion is stable around 10.00. Therefore, through the abovementioned comparative analysis, it can be known that among the three gas injection methods, the top gas injection method is most conducive to improving the condensate oil recovery of the Yaha condensate gas reservoir.

The final recovery factor histogram of different gas injection methods is shown in Figure 7.

It can be seen from Figure 7 that the recovery efficiency of condensate oil and condensate gas using the top gas injection method is the highest, with values of $80 \%$ and $71.71 \%$. The condensate recovery factor of bottom gas injection and shaft gas injection is basically the same, but the condensate recovery factor of bottom gas injection is slightly greater than that of shaft gas injection. Therefore, through comparative analysis, it can be known that the best gas injection 


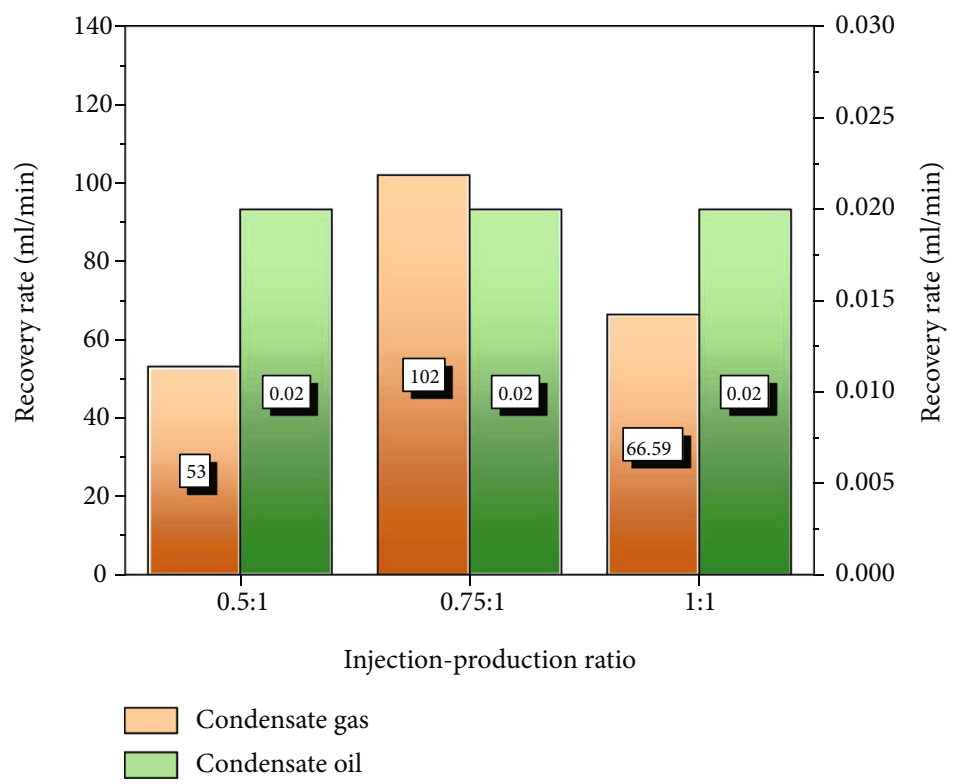

FIGURE 12: Histogram of the average recovery speed with different injection-production ratios.

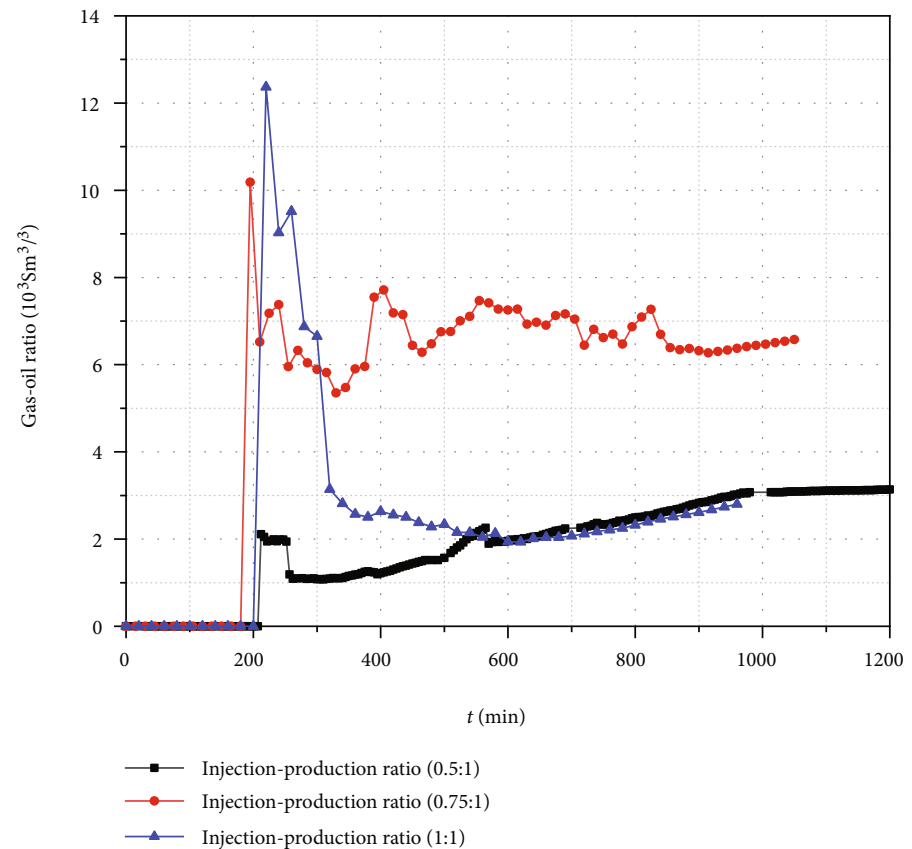

Figure 13: Production gas-oil ratio curves with different injection-production ratios.

method for the Yaha condensate gas reservoir is top gas injection.

4.2. Injection Medium. When studying the migration law of injected gas in the formation, it is necessary to consider the important factor of injected fluid characteristics. Because the physical properties of reservoir fluid and injected gas are quite different, the interaction between the two will seriously affect the migration of injected gas. Therefore, three gas injection media were selected for comparative analysis, and the injection media were $\mathrm{CH}_{4}, \mathrm{CO}_{2}$, and on-site gas.
The experimental gas injection method is shaft gas injection, and the injection-production ratio is $0.5: 1$. Other specific experimental parameters are shown in Table 3.

The average recovery rate of condensate gas and condensate oil for different gas injection methods is shown in Figure 8.

It can be seen from Figure 8 that for the average recovery rate of condensate gas, the effects of on-site gas injection and carbon dioxide injection are approximately the same, and both are much greater than methane gas injection. For the recovery rate of condensate oil, carbon dioxide injection 


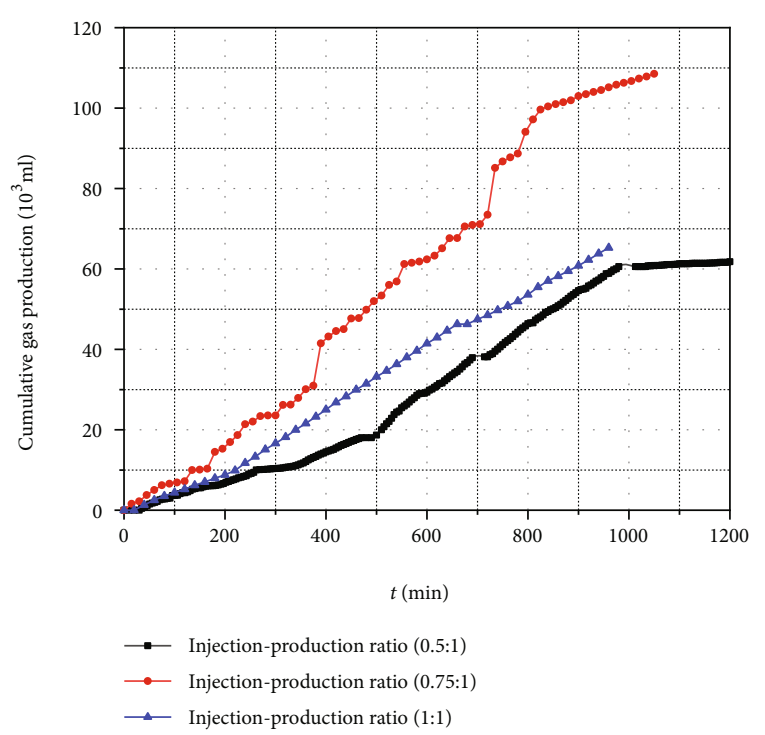

(a) Cumulative gas production curve

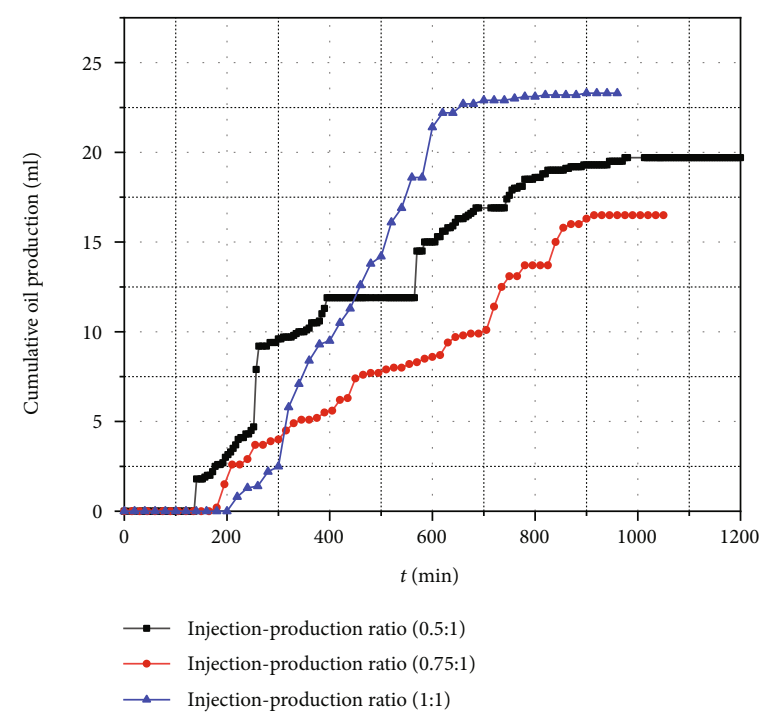

(b) Cumulative oil production curve

FIGURE 14: Cumulative production curve of different injection-production ratios.

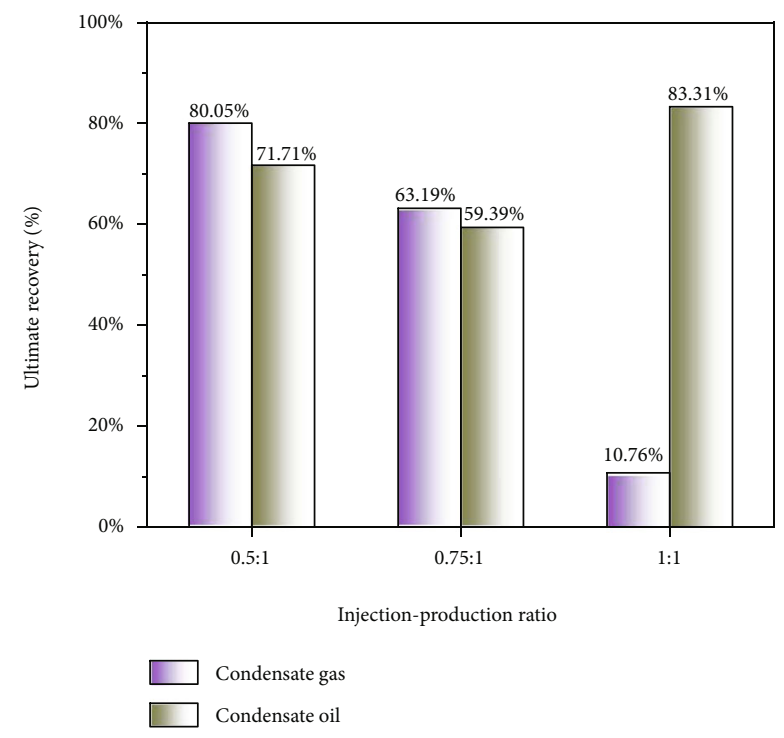

FIGURE 15: Histogram of the final recovery factor with different injection-production ratios.

has the best effect with a value of 0.03 , followed by on-site gas injection with a value of 0.02 , and finally methane injection with a value of 0.017 . Therefore, in order to improve the recovery of condensate gas and condensate oil, carbon dioxide injection is the best injection medium.

The production gas-oil ratio curves of different gas injection media are shown in Figure 9.

It can be seen from Figure 9 that the changing trends of the produced gas-oil ratio curves of different gas injection media are approximately the same, and they all show a trend of first rising rapidly and then falling and finally tending to stabilize. However, the production gas-oil ratio curve of carbon dioxide injection increased the most in the early stage, the maximum value was close to $8 \times 10^{3} \mathrm{Sm}^{3} \cdot \mathrm{m}^{-3}$, and finally stabilized near $3.7 \times 10^{3} \mathrm{Sm}^{3} \cdot \mathrm{m}^{-3}$. This is because it is affected by phase changes in the early stage of production, and even if condensate is precipitated in the later stage of production, the precipitated condensate is mixed with carbon dioxide, and the condensate is displaced by the mixed phase of carbon dioxide. On the whole, the average production gas-oil ratio of on-site gas injection is the largest, followed by carbon dioxide injection, and finally methane injection.

The cumulative production curves of condensate gas and condensate oil for different gas injection media are shown in Figure 10.

It can be seen from Figure 10(a) that under the same production pressure, the cumulative gas production curves of the three different gas injection methods are quite different, but they all show a trend of gradually increasing and then becoming stable. In the early stage of gas production $(t<500 \mathrm{~min})$, the gas production rate of on-site gas injection first increases and then decreases, while the gas production rate of carbon dioxide injection first decreases and then increases. And the cumulative gas production of on-site gas injection is greater than the cumulative gas production of carbon dioxide injection, and the gas production rate and cumulative gas production of methane gas injection are the smallest. In the middle stage of gas production $(t=500 \sim 800 \mathrm{~min})$, the gas production rate and cumulative gas production of carbon dioxide injection increase rapidly, which is much greater than the situation of on-site gas injection and methane gas injection. In the later stage of gas production $(t>800 \mathrm{~min})$, the gas production rate of carbon dioxide injection is still further increasing, so the cumulative gas production is also gradually increasing. However, the production of on-site gas and methane gas was gradually stopped. The cumulative production of on-site gas was finally stabilized at about $80 \times 10^{3} \mathrm{ml}$, and the cumulative 
production of methane gas was finally stabilized at about $60 \times 10^{3} \mathrm{ml}$.

It can be seen from Figure 10(b) that under the same production pressure, the cumulative oil production curves of the three different gas injection methods are quite different, but they all show a trend of increasing first and then becoming stable. In the early stage of oil production $(t<400 \mathrm{~min})$, the oil production rate and cumulative production of on-site gas injection are greater than those of carbon dioxide and methane injection. In the middle stage $(t=400 \sim 800 \mathrm{~min})$, the oil production rate and cumulative production of carbon dioxide injection gradually increase and eventually exceed the gas production rate and cumulative oil production of on-site gas injection. In the later stage $(t>800 \mathrm{~min})$, the output of carbon dioxide injection gradually reaches its maximum value, which is about $27.5 \mathrm{ml}$. The second is the cumulative oil production of on-site gas injection, while methane gas injection ends the oil production process earliest and the cumulative oil production is the lowest. Therefore, through the abovementioned comparative analysis, it can be known that among the three gas injection methods, carbon dioxide injection is most conducive to improving the recovery of condensate oil in the Yaha condensate gas reservoir, followed by on-site gas injection, and finally methane gas injection.

The final recovery factor histogram of different gas injection methods is shown in Figure 11.

It can be seen from Figure 11 that the final recovery efficiency of condensate gas and condensate oil under different gas injection medium conditions is more obvious. Among them, the production effect of carbon dioxide injection is the best. The final recoveries of condensate gas and condensate oil under this condition are both higher than $90 \%$, with values of $95.11 \%$ and $96.5 \%$, respectively. The final recovery efficiencies of methane injection and on-site gas injection are approximately the same. Therefore, in order to further improve the ultimate recovery of condensate gas and condensate oil, the most suitable gas injection medium should be selected.

4.3. Injection-Production Ratio. The injection volume of gas injection wells and production wells of condensate gas reservoirs directly affect the changes in gas layer pressure gradient, and pressure changes have a great impact on gas reservoir recovery. Therefore, a reasonable injectionproduction ratio is a key factor to ensure oil and gas recovery. In order to analyze the influence of injectionproduction ratio on the Yaha condensate gas reservoir, three physical simulation experiments of injection-production ratio were selected, which were $0.5: 1,0.75: 1$, and 1.1 . The experimental gas injection medium is methane, and the gas injection method is shaft gas injection. Other specific experimental parameters are shown in Table 3.

The average recovery rate of condensate gas and condensate oil for different gas injection-production ratios is shown in Figure 12.

It can be seen from Figure 12 that for the average recovery rate of condensate gas, when the injection ratio is $0.75: 1$, the average recovery rate is the largest, with a value of
$102 \mathrm{ml} \cdot \mathrm{min}^{-1}$. The second is the injection ratio of $0.5: 1$, and the last is the injection ratio of $1: 1$. For the recovery rate of condensate oil, the average recovery rate of the three injection-production ratios is the same, which is 0.02 .

The production gas-oil ratio curves of different gas injection-production ratios are shown in Figure 13.

It can be seen from Figure 13 that the changing trends of the produced gas-oil ratio curves of different gas injection media are approximately the same. The curves all show a rapid rise first, then fall, and finally stabilize. As the injection-production ratio increases, the production gas-oil ratio curve fluctuates more violently in the early production stage, and the peak value reached is higher. Through comparative analysis, it can be known that when the injectionproduction ratio is $1: 1$, the maximum gasoline production ratio is about $12.5 \times 10^{3} \mathrm{Sm}^{3} \cdot \mathrm{m}^{-3}$. In the later stages of production, the production gas-oil ratio curve with an injection-production ratio of $0.75: 1$ is significantly higher than the other two cases. The production gas-oil ratio curves with injection-production ratios of $0.5: 1$ and $1: 1$ are approximately coincident and are stable around $3.1 \times$ $10^{3} \mathrm{Sm}^{3} \cdot \mathrm{m}^{-3}$. Therefore, it can be seen that the production effect of the injection-production ratio of $0.5: 1$ and $1: 1$ is better than that of the injection-production ratio of $0.75: 1$.

The cumulative production curves of condensate gas and condensate oil with different gas injection ratios are shown in Figure 14.

It can be seen from Figure 14(a) that under the same production pressure, the cumulative gas production curves of the three different gas injection methods are quite different, but they all show an increasing trend. With the gradual extension of the production time, the gas production rate with an injection-production ratio of $0.75: 1$ is the fastest and the cumulative gas production always remains the largest, followed by the injection-production ratio $1: 1$. The gas production rate and cumulative gas production with an injection-production ratio of $0.5: 1$ is the lowest. Therefore, through comparative analysis, it can be known that the gas production effect with an injection-production ratio of $0.75: 1$ is the best.

It can be seen from Figure 14(b) that under the same production pressure, the cumulative oil production curves of the three different gas injection methods are quite different, but they all show a trend of increasing first and then becoming stable. In the early stage of oil production $(t<300 \mathrm{~min})$, the oil production rate and cumulative oil production with an injection-production ratio of $0.5: 1$ are greater than those of the other two groups. Moreover, the oil production rate and cumulative oil production with an injection-production ratio of $0.75: 1$ are greater than the case where the injection-production ratio is $1: 1$. In the middle and late stages $(t=400 \sim 800 \mathrm{~min})$, the oil production rate and cumulative oil production of $1: 1$ injection-production ratio gradually increase, and the final oil production stabilizes at around $23.3 \mathrm{ml}$. The cumulative oil production under this condition surpasses the other two injection-production ratios, which means that the condensate recovery effect is the best under this condition. 
The final recovery factor histogram of different gas injection methods is shown in Figure 15.

It can be seen from Figure 15 that as the injectionproduction ratio increases, the condensate gas recovery factor gradually decreases, while the condensate oil recovery factor gradually increases. When the injection-production ratio is $0.5: 1$, the recovery factor of condensate gas reaches its maximum value, which is $80.05 \%$. When the injectionproduction ratio is $1: 1$, the condensate recovery rate reaches the maximum value, which is $83.31 \%$. Therefore, through comparative analysis, it can be known that the increase of injection-production ratio is beneficial to the improvement of condensate oil recovery.

\section{Summary and Conclusions}

(1) Different gas injection methods have different effects on improving the recovery of condensate oil. The top gas injection has a high oil production rate and a low gas-oil ratio. When the production pressure is reduced from $58 \mathrm{MPa}$ to $39 \mathrm{MPa}$, oil is produced rapidly and the cumulative oil production is the largest. Therefore, under the same production conditions, top gas injection is the best choice, followed by shaft gas injection, and bottom gas injection has the worst effect

(2) The conclusions obtained from the comparative analysis of gas injection media are basically consistent with those obtained by previous scholars. When the pressure drops below the dew point pressure, the output of condensate increases sharply, the production effect of carbon dioxide injection is the best, and the condensate recovery rate reaches the maximum with a value of $96.5 \%$

(3) The higher of the injection-production ratio, the more stable the production and recovery rate of condensate oil and gas. The injection-production ratio and the recovery factor of condensate gas change in inverse proportion, and it is in direct proportion to the recovery factor of condensate oil. When the injection-production ratio is $1: 1$, the condensate recovery rate reaches the maximum value of $83.31 \%$

(4) The research in this paper is based on the Yaha condensate gas reservoir. Therefore, the conclusions obtained in this research can provide certain reference value and guiding significance for the injection parameters of similar gas reservoirs

\section{Data Availability}

The (data type) data used to support the findings of this study are available from the corresponding author upon request.

\section{Additional Points}

Highlights. (1) The Yaha gas condensate reservoir will appear reversed condensate in the later stage of exploitation.
(2) Indoor physical model research helps to understand the mechanism of gas migration. (3) Optimizing the condensate gas injection scheme helps to improve the recovery efficiency in the later stages of development.

\section{Conflicts of Interest}

The authors declare that they have no conflicts of interest.

\section{Acknowledgments}

This study was supported by the China Petroleum Major Science and Technology Project (Phase III) (No. 2018E1804.

\section{References}

[1] Z. Su, Y. Tang, H. J. Ruan, Y. Wang, and X. Wei, "Experimental and modeling study of $\mathrm{CO}_{2}$ - Improved gas recovery in gas condensate reservoir," Journal of Petroleum, vol. 3, no. 1, pp. 87-95, 2017.

[2] Z. Xie, H. Y. Deng, and W. Lei, "Study on phase characteristics of condensate gas reservoir with oil ring," Drilling \& Production Technology, vol. 32, no. 2, 2009.

[3] P. Guo and Z. C. Li, "Study on the phase behavior of formation fluid in Nanyishan-E3 condensate gas reservoir," Natural Gas Industry, vol. 19, no. 5, pp. 43-46, 1999.

[4] L. X. Zhou and X. B. Chu, "Study on the phase behavior of Fengshen-1 condensate gas reservoir," Journal of Southwest Petroleum University, vol. 30, no. 3, pp. 81-84, 2008.

[5] H. J. Lu, Study on the Distribution of Favorable Sedimentary Facies Belts in the Upper Paleozoic in Block Zhao 51 of Sulige Gas Field, Northwest University, Xi'an, 2014.

[6] R. Q. Ming, H. Q. He, and Q. F. Hu, "A new method for prediction of water breakthrough time in bottom water condensate gas reservoir," Special Oil and Gas Reservoirs, vol. 25, no. 5, pp. 99-103, 2018.

[7] R. Q. Ming, H. Q. He, and Q. F. Hu, “A new model for predicting water breakthrough time of high-yield wells in edge water condensate gas reservoirs," Special Oil and Gas Reservoirs, vol. 25, no. 2, pp. 76-79, 2018.

[8] Y. Tang, Z. M. Du, Z. L. Qi, S. L. Li, and L. Sun, "Current status and future development of the study on removal of near-wellbore damage in low-permeability gas condensate wells," Natural Gas Industry, vol. 27, no. 6, pp. 88-92, 2007.

[9] J. J. Sheng, "Increase liquid oil production by huff-n-puff of produced gas in shale gas condensate reservoirs," Journal of Unconventional Oil and Gas Resources, vol. 11, pp. 19-26, 2015.

[10] S. L. Li, Y. Pan, and L. Sun, "New ideas for improving the recovery efficiency of condensate gas reservoirs," Natural Gas Industry, vol. 28, no. 9, 2008.

[11] H. Lu, G. Ma, L. Cao, and M. Azimi, "Optimization of light hydrocarbon recovery system in condensate gas field," Energy Reports, vol. 5, pp. 1209-1221, 2019.

[12] M. Wang, S. Chen, and M. Lin, "Enhancing recovery and sensitivity studies in an unconventional tight gas condensate reservoir," Petroleum Science, vol. 15, no. 2, pp. 305-318, 2018.

[13] L. Jingsong, L. Xiangfang, K. Xiaodong, T. Min, and Z. Yongyi, "New method of cyclic gas injection for condensate gas reservoirs," Natural Gas Industry, vol. 24, pp. 76-79, 2004. 
[14] P. C. Dong, T. W. Jiang, M. L. Tang, and W. Xie, "Optimization of Yaha-5 condensate gas reservoir development method," Daqing Petroleum Geology and Development, vol. 26, no. 3, pp. 51-54, 2007.

[15] F. Lu, Gas injection enhanced oil recovery phase behavior and seepage mechanism of Dalaoba condensate gas reservoir, Journal of Southwest Petroleum University, Chengdu, 2019.

[16] C. W. Lu, "Optimize gas injection to improve the recovery rate of condensate gas field," Petrochemical Technology, vol. 23, no. 1, 2016.

[17] Y. L. Hu, B. Z. Li, and Z. D. Sun, "Selection of mining methods for condensate gas reservoirs," Natural Gas Geoscience, vol. 14, no. 5, pp. 398-401, 2003.

[18] J. Liu, Fine description of gas reservoirs in Wenjisang Gas Field, Northwest University, Xi'an, 2007.

[19] J. P. Shao and B. R. Niu, "Low-permeability condensate gas reservoir development technology," China Petroleum and Petrochemical, vol. 1, no. 1, pp. 62-63, 2007.

[20] C. F. Hu, K. Zheng, and X. P. Hu, “Application of reservoir numerical simulation technology in the development of condensate gas reservoirs with oil rings," Petroleum Geology and Engineering, vol. 24, no. 3, pp. 46-49, 2010.

[21] A. He, C. Zou, Y. Cui, J. Yan, H. Zhang, and Y. Tang, "Research on increasing the production of condensate with $\mathrm{CO}_{2}$ huff and puff in South A condensate gas reservoir of Zanarol Oilfield," Reservoir Evaluation and Development, vol. 10, no. 3, 2020.

[22] A. Hassan, M. Abdalla, M. Mahmoud, G. Glatz, A. al-Majed, and A. al-Nakhli, "Condensate-banking removal and gasproduction enhancement using thermochemical injection: a field-scale simulation," Processes, vol. 8, no. 6, p. 727, 2020.

[23] T. Wan and Z. J. Mu, "The use of numerical simulation to investigate the enhanced Eagle Ford shale gas condensate well recovery using cyclic $\mathrm{CO}_{2}$ injection method with nano-pore effect," Fuel, vol. 233, pp. 123-132, 2018.

[24] J. Jiang and R. M. Younis, "Compositional modeling of enhanced hydrocarbons recovery for fractured shale gascondensate reservoirs with the effects of capillary pressure and multicomponent mechanisms," Journal of Natural Gas Science and Engineering, vol. 34, pp. 1262-1275, 2016.

[25] Q. H. Feng, B. B. Deng, Y. Z. Yang, J. C. Zhang, X. H. Peng, and J. K. Yuan, "Reasonable and efficient gas injection development method for tight sandstone condensate gas reservoirs in Ordos Basin," Daqing Petroleum Geology and Development, vol. 39, no. 6, pp. 55-62, 2020.

[26] G. H. Yuan, R. J. Wang, S. G. Luo, L. Shang, and X. Z. Wang, "Laboratory test study on enhanced oil recovery by methane injection in highly saturated condensate gas reservoirs," Inner Mongolia Petrochemical Industry, vol. 34, no. 11, pp. 21-22, 2008.

[27] D. L. Hou, Study on the mechanism and storage of $\mathrm{CO}_{2}$ enhanced oil recovery in near-critical condensate gas reservoirs, Southwest Petroleum University, Chengdu, 2014.

[28] S. L. Sui, P. Guo, J. F. Du, and L. X. Xiao, "The experiment of gas condensate depletion in low-permeability porous medium," Journal of Southwest Petroleum University., vol. 32, no. 3, pp. 97-100, 2010. 